\title{
Assessment of the soil quality by fuzzy mathematics in farmland around a uranium mill tailing repository in China
}

\author{
X. Yan ${ }^{1}$ and X. Luo ${ }^{2, \star}$ \\ 1 College of Chemistry and Chemical Engineering, Qiqihar University Qiqihar, Heilongjiang 161006, P.R. China. \\ 2 Southwest University of Science and Technology Mianyang, Sichuan 621010, P.R. China.
}

Received 22 August 2015 - Accepted 16 October 2015

\begin{abstract}
Based on statistical analysis of the concentrations of multiple contaminants such as radionuclides, heavy metals and organochlorine pesticides in soil samples, the soil quality of a large piece of farmland around a uranium mill tailing (UMT) pond was assessed by fuzzy mathematics. This study found that the concentrations of $\mathrm{U}, \mathrm{Th}, \mathrm{Cd}, \mathrm{Pb}$ and DDT in all sampling sites and $\mathrm{Cr}$ in sites 1 and 2 were above the standard values, indicating the effects of the UMTs. We also detected residual OCPs in all soil samples in the study area. The fuzzy mathematics assessment showed that the farmland soil quality around the uranium mill tailing pond at site 1 belongs to class II, while sites $2-6$ belong to class I. Based on the above conclusions, in the present study most of the farmland soils were suitable for growing crops except for the areas near the UMT pond (0-500 meters).
\end{abstract}

Keywords: Uranium mill tailing / radionuclide / heavy metal / organochlorine pesticides / fuzzy mathematics

\section{Introduction}

In a natural environment, there are a variety of pollutants in the soils such as acid, alkali, heavy metals, salts, radionuclides, OCPs, phenols, cyanides, oils and synthetic detergents (Yang et al., 2014). In South China, there is a UMT repository which contains approximately $2 \times 10^{8}$ t of UMTs. The UMTs contain a series of long-lived radionuclides, such as uranium (U) and thorium (Th). Also, the UMTs contain some associated heavy metals such as chromium $(\mathrm{Cr})$, cadmium $(\mathrm{Cd})$, copper $(\mathrm{Cu})$, zinc ( $\mathrm{Zn}$ ) and lead $(\mathrm{Pb})$ (Yan and Luo, 2015). Around the UMT pond, there is a piece of farmland (900 ha) which is one of the local crop production areas. Over time, the farmland soils will receive radionuclides and heavy metals discharged from the UMTs and organochlorine pesticides such as hexachlorocyclohexane (HCH) and dichlorodiphenyl trichloroethane (DDT) applied by local farmers. Therefore, it is necessary to investigate the pollution status of the farmland soils and give an objective assessment of the soil environmental quality. Fuzzy mathematics methods comprehensively evaluate the contributions of various pollutants according to predetermined weights, and decrease the fuzziness by using membership functions. Therefore, the sensitivity is quite high compared with other index evaluation techniques such as Bayesian statistics (Onkal-Engin et al., 2004). In the present study, the objectives of this paper were: (1) to investigate the pollution status of the farmland soils, and (2) to assess the current situation of the soil environmental quality in the farmland around the UMT

\footnotetext{
^m13836295186@163.com
}

pond by applying fuzzy mathematics assessment according to the concentrations of radionuclides ( $U$ and $\mathrm{Th}$ ), heavy metals $(\mathrm{Cr}, \mathrm{Cd}, \mathrm{Cu}, \mathrm{Zn}$ and $\mathrm{Pb}$ ) and OCPs (DDT and $\mathrm{HCH}$ ) in the soils.

\section{Materials and methods}

\subsection{Analyses of soil samples}

Six individual soil samples were collected from a farmland cultivated with Chinese cabbage and watermelon at a distance of $100 \mathrm{~m}$ (S1), $500 \mathrm{~m}(\mathrm{~S} 2), 900 \mathrm{~m}$ (S3), $1300 \mathrm{~m}$ (S4), $1700 \mathrm{~m}$ (S5) and $2100 \mathrm{~m}$ (S6) from the UMT repository with 10 replications in each sampling site (Figure 1). The sampling depth was $0-15 \mathrm{~cm}$. The soil is a clayey yellow soil which is an Ultisol in US soil taxonomy, with $71.27 \%$ clay, $10.15 \%$ silt and $17.25 \%$ sand. Collected soil samples were packed into a water-tight bag to prevent cross-contamination and shipped to the laboratory. In the laboratory, the soil samples were kept drying at room temperature for 4 weeks, then they were sealed in a clean polyethylene container and stored in a refrigerator for analysis of the total concentrations of radionuclides ( $U$ and $\mathrm{Th}$ ), heavy metals ( $\mathrm{Cr}, \mathrm{Cd}, \mathrm{Cu}, \mathrm{Zn}$ and $\mathrm{Pb}$ ) and OCPs (DDT and $\mathrm{HCH}$ ). For each soil sample, $0.100 \mathrm{~g}$ of soil sample was taken and digested with analytical reagent-grade $\mathrm{HNO}_{3}$ and $\mathrm{HF}$, which were purified by sub-boiling distillation to give a low reagent blank and a corresponding low analytic limit of detection. The concentrations of radionuclides and heavy metals in soil samples were determined by inductively coupled 
plasma mass spectrometry (SPECTRO MS, Germany). The concentration of OCPs in soil samples was determined by gas chromatography (GC 2008B, China) (Jamal, 2011).

\subsection{Fuzzy comprehensive assessment}

Nine elements (U, Th, $\mathrm{Cr}, \mathrm{Cd}, \mathrm{Cu}, \mathrm{Zn}, \mathrm{Pb}, \mathrm{DDT}$ and $\mathrm{HCH}$ ) in farmland soils are selected as evaluation indicators $(\chi)$. The assessment criteria are established from the local background values, the environmental chemistry of elements and the environmental quality standard for soils of China (GB 156181995) (Bowen, 1979; Cai et al., 2006). The soil quality can be classified on five levels: class I, clean; class II, ordinary; class III, light pollution; class IV, moderate pollution and V, intense pollution. For example, if the soil quality is classified as class IV (moderate pollution) or V (intense pollution), it means the extent of soil contamination is similar to that of uranium mill tailings, and most plants cannot grow normally in the soils except for invasive plants and hyperaccumulators. If the soil quality is classified as class III (light pollution), it means most plants can grow normally. However, the radionuclide $\mathrm{U}$ will present a health hazard through the food chain. If the soil quality is classified as class II (ordinary), it means a small amount of the residual radionuclide $\mathrm{U}$ will exist in plants. If the soil quality is classified as class I (clean), it means the radionuclide $\mathrm{U}$ has no effect on plant growth.

The membership degrees of assessment parameters at each level can be described quantitatively by a set of formulae of membership functions as follows: membership functions of certain elements in the first to fifth level soil are

$$
\begin{aligned}
& \varphi_{1}(\chi)=\left\{\begin{array}{l}
1 \chi \leqslant S 1 \\
(S 2-\chi) /(S 2-S 1) S 1<\chi<S 2 \\
0 \chi \geqslant S 2
\end{array}\right. \\
& \varphi_{2}(\chi)=\left\{\begin{array}{l}
0 \chi \leqslant S 1 \\
1-\varphi 1(\chi) S 1<\chi<S 2 \\
(S 3-\chi) /(S 3-S 2) S 2 \leqslant \chi<S 3 \\
0 \chi \geqslant S 3
\end{array}\right. \\
& \varphi_{3}(\chi)=\left\{\begin{array}{l}
0 \chi \leqslant S 2 \\
1-\varphi 2(\chi) S 2<\chi<S 3 \\
(S 3-\chi) /(S 3-S 2) S 3 \leqslant \chi<S 4 \\
0 \chi \geqslant S 4
\end{array}\right. \\
& \varphi_{4}(\chi)=\left\{\begin{array}{l}
0 \chi \leqslant S 3 \\
1-\varphi 3(\chi) S 3<\chi<S 4 \\
(S 3-\chi) /(S 3-S 2) S 4 \leqslant \chi<S 5 \\
0 \chi \geqslant S 5
\end{array}\right. \\
& \varphi_{5}(\chi)=\left\{\begin{array}{l}
0 \chi \leqslant S 4 \\
(\chi-S 4) /(S 5-S 4) S 4<\chi<S 5 \\
1 \chi \geqslant S 5 .
\end{array}\right.
\end{aligned}
$$

$\varphi_{\mathrm{j}}(\chi)$ is the membership degree of assessment parameter $(\chi)$ in class I, II, III, IV and V, respectively. Substitute the measured

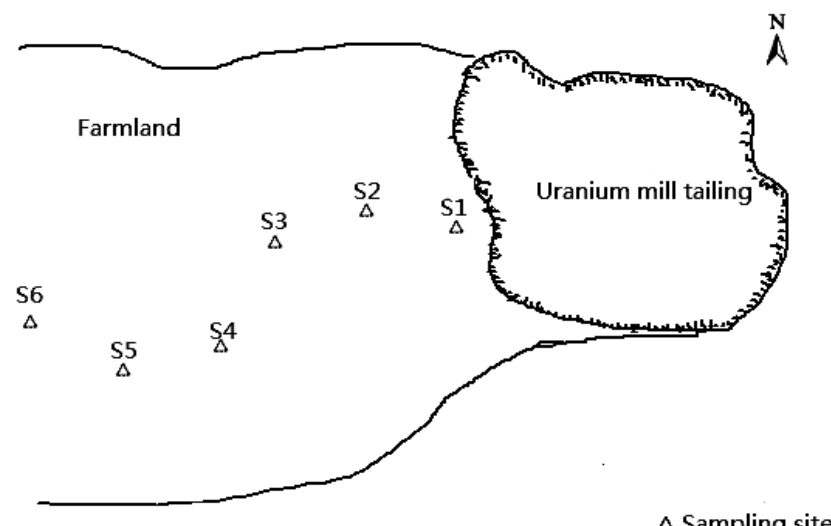

Figure 1. Map of the sampling sites for soil samples.

data of each assessment parameter at each sampling site and the standard values into the membership functions. Then, we can obtain the fuzzy matrix $\tilde{\mathrm{A}}$, which can be expressed as:

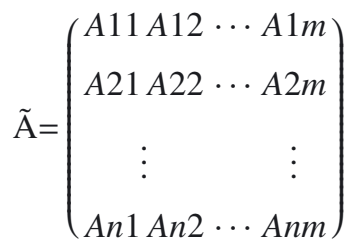

where $A_{i j}(i=1,2 \ldots n ; j=1,2 \ldots m)$ is the membership degree of the $i$ th assessment parameter at the $j$ th level. Here, $n$ is 9 and $m$ is 5 . Allocate the weights of each assessment parameter at each sampling site to obtain the matrix õ with the formula $W_{i(k)}=\left(\chi_{i(k)} / s_{i}\right) / \sum_{\mathrm{i}=1}^{\mathrm{n}}\left(\chi_{i(k)} / s_{i}\right)$. Here, $\chi_{i(k)}$ is the measured concentration of the $i$ th assessment parameter at the $k$ th monitoring site, $s_{i}$ is the average assessment criteria of the $i$ th assessment parameter, and $W_{i(k)}$ means the weight of the $i$ th assessment parameter at the $k$ th monitoring site. $\tilde{\mathrm{O}}_{(k)}$, the weight matrix $\tilde{O}$ at the monitoring site $k$, can be expressed as $\tilde{\mathrm{O}}_{(k)}=\left(W_{1(k)}, W_{2(k)} \ldots W_{n(k)}\right)$. Here, $k$ is 6 . The fuzzy algorithm of $\tilde{O} \cdot \tilde{A}$ can be computed by matrix multiplication. This method is described as follows: Weight matrix $\tilde{\mathrm{O}}=\left(W_{i}\right)_{1 \times n}$ and Fuzzy matrix $\tilde{\mathrm{A}}=\left(A_{i j}\right)_{n \times m}$. Then, the assessment results can be obtained: $\tilde{\mathrm{O}} \cdot \tilde{\mathrm{A}}=\left(O_{1}, O_{2} \ldots\right.$ Om $)$ (Shen et al., 2005).

The Microsoft Excel program package was used to statistically evaluate the data obtained in the experiments. Pearson's correlation coefficient was calculated using SPSS 19.0.

\section{Results and discussions}

The concentrations of radionuclides, heavy metals and OCPs of farmland soils around the UMT repository are presented in Table 1. The concentrations of the radionuclides $U$ and $\mathrm{Th}$ in all sites, and the heavy metals $\mathrm{Cr}$ in site 1 and site 2, and $\mathrm{Cd}$ and $\mathrm{Pb}$ in all sites were above the standard values (Bowen, 1979; Cai et al., 2006). We also found that all the radionuclides and heavy metals were distributed such that the contents decreased with the increase in distance from the UMT pond except for $\mathrm{Cd}$ and $\mathrm{Pb}$. The above results indicate that the 
Table 1. The concentrations of radionuclides, heavy metals and OCPs of analyzed farmland soil samples (Mean $\pm \mathrm{SD})\left(\mathrm{mg} \mathrm{kg} \mathrm{g}^{-1}\right)$.

\begin{tabular}{cccccccccc} 
Samples & $\mathbf{U}$ & Th & $\mathbf{C d}$ & $\begin{array}{c}\mathbf{C r} \\
\left(\times \mathbf{1 0}^{\mathbf{3}}\right)\end{array}$ & $\mathbf{C u}$ & $\mathbf{Z n}$ & $\mathbf{P b}$ & $\begin{array}{c}\text { HCH } \\
\left(\times \mathbf{1 0}^{-\mathbf{3}}\right)\end{array}$ & $\begin{array}{c}\text { DDT } \\
\left(\times \mathbf{1 0}^{-\mathbf{3}}\right)\end{array}$ \\
\hline S1 & $12.35 \pm 0.52$ & $16.7 \pm 1.36$ & $0.24 \pm 0.03$ & $0.14 \pm 0.01$ & $7.09 \pm 0.55$ & $18.8 \pm 1.76$ & $50.7 \pm 3.46$ & $16.4 \pm 1.57$ & $97.5 \pm 16.1$ \\
S2 & $11.91 \pm 1.09$ & $15.2 \pm 0.75$ & $0.23 \pm 0.04$ & $0.12 \pm 0.01$ & $6.12 \pm 0.49$ & $17.4 \pm 0.97$ & $51.4 \pm 3.27$ & $17.4 \pm 1.70$ & $98.1 \pm 10.8$ \\
S3 & $9.43 \pm 0.13$ & $14.3 \pm 0.93$ & $0.25 \pm 0.03$ & $0.08 \pm 0.01$ & $5.57 \pm 0.31$ & $17.1 \pm 1.28$ & $48.9 \pm 3.87$ & $16.4 \pm 3.32$ & $91.8 \pm 13.7$ \\
S4 & $7.13 \pm 0.39$ & $14.1 \pm 0.65$ & $0.22 \pm 0.03$ & $0.06 \pm 0.008$ & $4.58 \pm 0.29$ & $16.3 \pm 1.11$ & $48.8 \pm 4.01$ & $16.6 \pm 2.84$ & $98.6 \pm 15.2$ \\
S5 & $5.54 \pm 0.25$ & $14.1 \pm 0.95$ & $0.22 \pm 0.03$ & $0.06 \pm 0.006$ & $4.14 \pm 0.13$ & $14.0 \pm 1.28$ & $46.5 \pm 3.79$ & $17.5 \pm 2.46$ & $101.5 \pm 14.1$ \\
S6 & $5.21 \pm 0.26$ & $13.2 \pm 0.53$ & $0.22 \pm 0.03$ & $0.04 \pm 0.003$ & $3.89 \pm 0.27$ & $11.1 \pm 1.05$ & $45.5 \pm 2.97$ & $16.2 \pm 2.05$ & $93.0 \pm 14.1$ \\
SV $^{*}$ & 2.00 & 12.00 & 0.20 & 0.09 & 35 & 100 & 35 & 50 & 50 \\
\hline
\end{tabular}

*SV: standard values $=$ class I.

Table 2. Correlation coefficients between soil pollutants.

\begin{tabular}{cccccccccc}
\hline & $\mathbf{U}$ & Th & $\mathbf{C d}$ & $\mathbf{C r}$ & $\mathbf{C u}$ & $\mathbf{Z n}$ & $\mathbf{P b}$ & $\mathbf{H C H}$ & $\mathbf{D D T}$ \\
\hline $\mathrm{U}$ & 1 & $0.66^{* *}$ & 0.24 & $0.91^{* *}$ & $0.90^{* *}$ & $0.79^{* *}$ & $0.48^{* *}$ & 0.06 & -0.003 \\
$\mathrm{Th}$ & & 1 & $0.27^{*}$ & $0.77^{* *}$ & $0.74^{* *}$ & $0.62^{* *}$ & 0.24 & -0.04 & -0.02 \\
$\mathrm{Cd}$ & & & 1 & 0.16 & $0.30^{*}$ & $0.28^{*}$ & 0.16 & -0.14 & 0.06 \\
$\mathrm{Cr}$ & & & & 1 & $0.90^{* *}$ & $0.75^{* *}$ & $0.43^{* *}$ & 0.02 & -0.01 \\
$\mathrm{Cu}$ & & & & & 1 & $0.76^{* *}$ & $0.44^{* *}$ & -0.04 & -0.01 \\
$\mathrm{Zn}$ & & & & & 1 & $0.50^{* *}$ & -0.02 & 0.07 \\
$\mathrm{~Pb}$ & & & & & & 1 & -0.12 & 0.13 \\
$\mathrm{HCH}$ & & & & & & & 1 & 0.10 \\
$\mathrm{DDT}$ & & & & & & & & & 1 \\
\hline
\end{tabular}

${ }^{*} P<0.05 ;{ }^{* *} P<0.01$.

farmland soils in the study area were affected by outside pollutants apart from the UMTs. In fact, the discharged radionuclides and associated heavy metals from the UMTs might be due to the uranium ore grade, mining technology, management measures of the UMTs, the damage to the soil cover and artificial disturbance. Through field research in uranium mines, Tripathi et al. (2008) found that the concentration of $\mathrm{U}$ in UMTs was 392-405 mg kg-1. Mangset and Sheyin (2009) collected a total of thirty-one UMT samples and found that the concentration of Th was $3900-4200 \mathrm{mg} \mathrm{kg}^{-1}$. Based on these analyses, if no appropriate measures such as increasing soil cover and setting up isolated areas are taken, the degree of soil pollution will be more serious. In the present study, our data showed that the concentration of $\mathrm{HCH}$ was $0.016 \mathrm{mg} \mathrm{kg}^{-1}$ and that of DDT was $0.098 \mathrm{mg} \mathrm{kg}^{-1}$, which indicated that both $\mathrm{HCH}$ and DDT remained in soils in the study area. We can also see that the concentration of DDT in all sampling sites was more than the environmental quality standard for soils of China (GB 15618-1995), which indicated the farmland soils were contaminated by OCPs, especially DDT. In recent years, new requests for food safety have been put forward. Therefore, some improvement measures such as changing traditional cultivation techniques need to be carried out in the study area.

To identify to what extent these elements can exert an influence together on the study area, correlation analyses were carried out between element pairs (Table 2). The results of the correlative analysis showed that combined pollution exists in the soil. Through the correlation analysis, the concentrations of radionuclides were significantly correlated with the concentration of heavy metals in the study area. It indicated that these pollutants are assumed to originate from the same pollution source (UMTs). However, the concentrations of $\mathrm{HCH}$ and
DDT were less related to the radionuclides and heavy metals, which suggested that these pollutants are assumed to originate from a different source such as the application of chemical pesticides by local farmers.

In the present study, the soil pollutants $\mathrm{U}, \mathrm{Th}, \mathrm{Cd}, \mathrm{Cr}$, $\mathrm{Cu}, \mathrm{Zn}, \mathrm{Pb}, \mathrm{HCH}$ and DDT were selected as assessment parameters to form an assessment factor, based on the farmland soil measured data in the farmland around the UMT repository. An assessment criterion was also established according to the local background values, the environmental chemistry of elements and the environmental quality standard for soils of China (GB 15618-1995). According to the formulae of the membership function, the membership degrees of each assessment parameter can be calculated at class I, II, III, IV and V (Table 3). After substitution of the actual measured data and standards at each level, we obtain fuzzy matrices of $\tilde{\mathrm{A}}_{1}-\tilde{\mathrm{A}}_{6}$ and weight matrices $\widetilde{\mathrm{O}}_{1}-\widetilde{\mathrm{O}}_{6}$. For example, at site 1 , the averages of the assessment criteria for $\mathrm{U}, \mathrm{Th}, \mathrm{Cd}$, $\mathrm{Cr}, \mathrm{Cu}, \mathrm{Zn}, \mathrm{Pb}, \mathrm{HCH}$ and DDT were $14,32,0.582,198,165$, 260, 221, 0.39 and 0.39 , respectively. The measured concentrations of these elements at site 1 were $12.35,16.7,0.24,140$, $7.09,17.4,18.8,0.016$ and $0.098 \mathrm{mg} \mathrm{kg}^{-1}$, respectively. So, $W_{1(1)}=\frac{12.35 / 14}{\frac{12.35}{14}+\frac{16.7}{32}+\frac{0.24}{0.52}+\frac{140}{108}+\frac{7.09}{165}+\frac{18.8}{260}+\frac{50.7}{221}+\frac{0.016}{0.39}+\frac{0.098}{0.39}}=\frac{0.88}{3.15}=0.28 ;$ $W_{2(1)}=\frac{0.52}{3.15}=0.17 ; W_{3(1)}=\frac{0.41}{3.15}=0.13 ; W_{4(1)}=\frac{0.71}{3.15}=0.23 ;$ $W_{5(1)}=\frac{0.04}{3.15}=0.01 ; W_{6(1)}=\frac{0.07}{3.15}=0.02 ; W_{7(1)}=\frac{0.23}{3.15}=0.07$; $W_{8(1)}=\frac{0.04}{3.15}=0.02 ; W_{9(1)}=\frac{0.25}{3.15}=0.08$. Then we obtain $\tilde{\mathrm{O}}_{1}=(0.28,0.17,0.13,0.23,0.01,0.02,0.07,0.02,0.08)$. Similarly, we can also obtain $\tilde{\mathrm{O}}_{2}=(0.29,0.16,0.13,0.21$, $0.01,0.02,0.08,0.01,0.08) ; \tilde{\mathrm{O}}_{3}=(0.26,0.18,0.17,0.16,0.01$, $0.03,0.09,0.02,0.09) ; \tilde{\mathrm{O}}_{4}=(0.24,0.20,0.18,0.14,0.01,0.03$, $0.10,0.01,0.09) ; \tilde{\mathrm{O}}_{5}=(0.21,0.21,0.18,0.14,0.009,0.02$, 
$0.10,0.02,0.12) ; \widetilde{\mathrm{O}}_{6}=(0.19,0.21,0.20,0.10,0.01,0.02,0.11$, $0.02,0.13)$.
Table 3. Standards for radionuclides, heavy metals and OCPs in soil $\left(\mathrm{mg} \mathrm{kg}^{-1}\right)$.

\begin{tabular}{cccccc}
\hline Parameters & I & II & III & IV & V \\
\hline $\mathrm{U}$ & 2 & 8 & 14 & 20 & 26 \\
$\mathrm{Th}$ & 12 & 22 & 32 & 42 & 52 \\
$\mathrm{Cd}$ & 0.2 & 0.4 & 0.8 & 1.0 & 1.5 \\
$\mathrm{Cr}$ & 90 & 150 & 200 & 250 & 300 \\
$\mathrm{Cu}$ & 35 & 50 & 140 & 200 & 400 \\
$\mathrm{Zn}$ & 100 & 160 & 220 & 320 & 500 \\
$\mathrm{~Pb}$ & 35 & 70 & 200 & 300 & 500 \\
$\mathrm{HCH}$ & 0.05 & 0.1 & 0.3 & 0.5 & 1.0 \\
\hline $\mathrm{DDT}$ & 0.05 & 0.1 & 0.3 & 0.5 & 1.0 \\
\hline
\end{tabular}

The fuzzy algorithms of Õ. $\tilde{A}$ gave the following results:

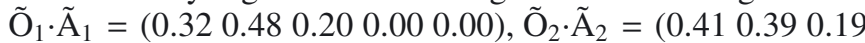

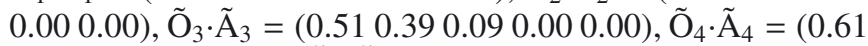

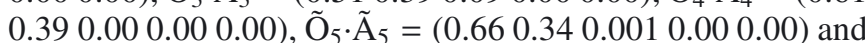

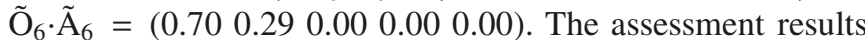

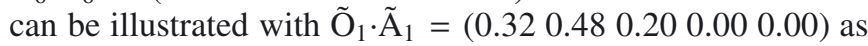
an example. Since the membership degree of class II $(0.48)$ is higher than that of class I (0.32), class III (0.20), class IV $(0.00)$ and class $\mathrm{V}(0.00)$, the soil environmental quality at site 1 belongs to class II. In addition, trends in soil environment quality changes at different sites varied. This can also be illustrated by the variations between membership degrees at different levels. The less difference there was, the greater the trend toward the next class. Hence, this trend at site 1 is more prominent than that at the other sites, suggesting that this area was most seriously affected by the UMTs. All these results indicate that it is not very suitable for growing high-quality crops within 500 meters (especially 100 meters) of the UMT pond. In the same way, we can conclude that the environmental quality of the soil at sites 2-6 belongs to class I. In general, these values indicate that it is relatively safe for crop production.

\section{Conclusions}

Three conclusions may be drawn from this study: (1) the concentrations of all the radionuclides and most of the heavy metals in the soils around the UMT repository are above the standard values. This means extraneous pollutants have been added to the soil since the UMT decommissioning; (2) in the present study, OCPs were detectable in all soil samples, especially DDT; (3) on account of the measured values, the fuzzy mathematics assessment method provides a scientific basis for analyzing and evaluating the environmental quality of farmland soil. According to the local background values, the environmental chemistry of elements and the environmental quality standard for soils of China (GB 15618-1995), the soil around the uranium mill tailing repository at site 1 belongs to class II, while sites $2-6$ belong to class I.

Acknowledgements. This study was supported by the National Nuclear Facilities Decommissioning and Radioactive Waste Management key scientific research projects of China (No. 14ZG6101). We wish to express our warm thanks to the Engineering Research Center 
of Biomass Materials, Ministry of Education, Southwest University of Science and Technology.

\section{References}

Bowen H.J.M. (1979) Environmental chemistry of elements. Academic Press, New York.

Cai Y.M., Liu F.Z., Wang Y.H., Shi R.G., Liu M., Liu B.F. (2006) Discussion About Soil Environmental Quality Standard, China Journal of Agro-Environment Science 1, 32-35.

Jamal H.M. (2011) Occurrence of organochlorine pesticide residues in groundwater and soil from Syrian coastal area, Agriculture $\mathcal{E}$ Biology Journal of North America 2, 488-492.

Mangset W., Sheyin A. (2009) Measurement of radionuclides in processed mine tailings in Jos, Plateau State Bayero, J. Pure Appl. Sci. 2, 56-60.
Onkal-Engin G., Demir I., Hiz H. (2004) Assessment of urban air quality using fuzzy synthetic evaluation, Atmospheric Environment 38, 3809-3815.

Shen G., Lu Y., Wang M., Sun Y. (2005) Status and fuzzy comprehensive assessment of combined heavy metal and organo-chlorine pesticide pollution in the Taihu Lake region of China, J. Environ. Manage. 76, 355-362.

Tripathi R., Sahoo S., Jha V., Khan A., Puranik V. (2008) Assessment of environmental radioactivity at uranium mining, processing and tailings management facility at Jaduguda, India, Appl. Radiat. Isotopes 66, 1666-1670.

Yan X., Luo X. (2015) Radionuclides distribution, properties, and microbial diversity of soils in uranium mill tailings from southeastern China, J. Environ. Radioact. 139, 85-90.

Yang H., Huang X., Thompson J.R., Flower R.J. (2014) China's soil pollution: Urban brownfields, Science 344, 691-692.

Cite this article as: X. Yan, X. Luo. Assessment of the soil quality by fuzzy mathematics in farmland around a uranium mill tailing repository in China. Radioprotection 51(1), 37-41 (2016). 